\title{
Effect of Endometrioma cystectomy on cytokines of follicular fluid and IVF outcomes
}

\author{
Yu Liang, Xiaokui Yang, Yonglian Lan, Lingling Lei, Ying Li ${ }^{*}$ and Shuyu Wang ${ }^{*}$ (D)
}

\begin{abstract}
Background: Endometriosis patients undergoing in vitro fertilization-embryo transfer (IVF-ET) treatment suffer from lower success rates. The success of IVF-ET is related to the receptivity of the uterus and the quality of embryos, and it is well known a patient's endometriosis does not impair the receptivity. Whether endometrioma should be removed surgically before IVF remains controversial. Studies have shown that endometrioma removal decreases peritoneal inflammation, but little information is available regarding the alteration in the cytokines of follicular fluid. The objective of this study was to examine the impact of endometrioma cystectomy on the outcome of IVF and the levels of intrafollicular inflammatory cytokines and to investigate correlations between cytokine concentrations and IVF outcomes.
\end{abstract}

Method: A total of 41 women with endometriosis-associated infertility undergoing IVF were recruited; 13 patients (surgery group, $\mathbf{S}$ group) had surgery to remove the endometrioma before enrollment, and 28 patients (non-surgery group, NS group) were untreated before IVF. The follicular fluid from a dominant follicle was collected during oocyte retrieval, and the concentrations of sixteen soluble cytokines known to be involved in ovarian function were measured.

Results: Among the soluble molecules examined in this study, chemokines and growth factors and a few are inflammatory cytokines were found in the follicular fluid of patients with endometriosis. In addition, the expression levels of chemokines, growth factors, and most inflammatory cytokines did not differ between the $\mathrm{S}$ and NS groups, but interleukin (IL)-18 levels were significantly lower in the NS group. However, the levels of IL-18 in the FF did not correlate with IVF cycle parameters. The implantation and clinical pregnancy rates were similar between the two groups, but the antiMüllerian hormone (AMH) level was lower in the S group than in the NS group.

Conclusions: These findings suggest that endometrioma surgery may potentially reduce the ovarian reserve and has little impact on the success rate of IVF. Ovarian endometriomas are not associated with cytokine profiles in FF from infertile women, and they are not likely to affect the quality of the oocyte and embryo as a result of an inflammatory mechanism.

Keywords: Endometriosis, IL-18, Ovarian endometrioma, Follicular fluid, Infertility, In vitro fertilization (IVF)

\footnotetext{
*Correspondence: liying200306@126.com; myteacher1957@163.com Department of Human Reproductive Medicine, Beijing Obstetrics and Gynecology Hospital, Capital Medical University, Beijing 100026, China
}

(c) The Author(s). 2019 Open Access This article is distributed under the terms of the Creative Commons Attribution 4.0 International License (http://creativecommons.org/licenses/by/4.0/), which permits unrestricted use, distribution, and reproduction in any medium, provided you give appropriate credit to the original author(s) and the source, provide a link to the Creative Commons license, and indicate if changes were made. The Creative Commons Public Domain Dedication waiver (http://creativecommons.org/publicdomain/zero/1.0/) applies to the data made available in this article, unless otherwise stated. 


\section{Introduction}

Endometriosis is a chronic gynecological disorder affecting more than $10 \%$ of women of reproductive age [1]. It is defined as the presence of endometrial glands and stroma outside the uterine cavity, causing chronic pelvic pain, dysmenorrheal, and infertility [2]. Endometriosis is strongly associated with infertility; several studies suggest that 20 to $50 \%$ of infertile women suffer from endometriosis and that 30 to $50 \%$ of women with endometriosis experience impaired fertility [3, 4]. Although several conditions have been associated with the occurrence of endometriosis, including anatomic distortion, decreased oocyte quality and impaired endometrial receptivity, the mechanisms of endometriosis-associated infertility are not fully understood [5].

It is widely accepted that endometriosis is a chronic inflammatory disease. Many studies have demonstrated that endometriomas lead to the recruitment of immune cells and an intense inflammatory response, with increased levels of proinflammatory cytokines, growth factors, and angiogenesis [6-8]. Jørgensen et al. (2017) measured a panel of 48 different cytokines from the peritoneal fluid of infertile patients and identified 13 cytokines that discriminate for the presence or absence of endometriosis [9]. These cytokines can be broadly considered as belonging to three subgroups with overlapping biological functions: chemokines (IL-8, MCP-1, MCP-3, and CTACK), hematopoietic growth factors (IL5, IL-13, IL-9, M-CSF, and G-CSF), and general growth factors (HGF and LIF) [9]. Endometriosis is characterized by the increased number and activation of peritoneal macrophages and reduced cytotoxic effect of natural killer cells [10] and reactivity of $\mathrm{T}$ lymphocytes [11]. The increased number of activated peritoneal macrophages produce higher levels of pro-inflammatory cytokines, such as tumor necrosis factor- $\alpha$, IL- 6 , and IL-1 $\beta$. The reduced cytotoxic effect of NK cells can increase autoimmune reactivity, while the decreased $\mathrm{T}$ cell reactivity changes the Th1/Th2 balance toward Th2. Imbalanced Th1/Th2 may be related to endometriosis-associated infertility [12].

A damaging inflammatory milieu has also been proposed as a cause of diminished oocyte quality, which in turn could lead to poor IVF outcomes in patients with endometriosis [13, 14]. Follicular fluid [9] forms the biochemical micro-environment of the oocyte before ovulation and is used for estimating the developmental competence of female gametes [15]. The microenvironment of follicular fluid (FF) is closely associated with the formation of spindles and the distribution of chromosomes [16]. Singh et al. (2016) found a significant increase in the levels of proinflammatory (IL-1 $\beta$, TNF- $\alpha$, IL-2, IL-8, IL-12, and IFN- $\gamma$ ) and anti-inflammatory (IL-4, IL-6, and IL-10) cytokines in FF from women with endometriosis undergoing in vitro fertilization (IVF) as compared with controls [13]. Sarapik et al. (2012) found that IL-12 levels were positively correlated with oocyte fertilization and embryo development, while increased IL-18, IL-8, and MIP-1 $\beta$ levels were associated with successful IVF-induced pregnancy [17].

Endometriosis patients undergoing IVF treatment typically have a low success rate for establishing pregnancy [18-20]. The success of the IVF-ET process is related to the receptivity of the uterus and high-quality embryos, and it is well known that endometrial receptivity in eutopic endometrium in patients with endometriosis is not affected [21]. Evidence of the impact of an endometrioma on IVF is equivocal; the question of whether or not endometriomas should be surgically removed before IVF remains controversial [22, 23]. Previous studies have shown that endometriotic lesion removal decreases peritoneal inflammation [24], but little information is available regarding alterations of the intrafollicular inflammatory cytokine system. Because various cytokines in FF have been shown to play an important role in oocyte quality, including inflammatory factors, chemokines, and growth factors, it is important to investigate the impact of surgery on these cytokines.

The objective of this study was to examine the impact of endometrioma excision on the outcome of IVF and the levels of intrafollicular inflammatory cytokines in women with endometriosis, as well as to investigate correlations between cytokine concentrations and IVF outcomes.

\section{Materials and methods \\ Patients}

This prospective study was conducted between March 2017 and October 2018 at the Department of Human Reproductive Medicine, Beijing Obstetrics and Gynecology Hospital, Capital Medical University. Ethical approval was received from the local Institutional Ethics Committee and written informed consent was obtained from all participants. Women attending the center with indications for IVF treatment were recruited.

Forty-one patients with infertility due to stage III or IV endometriosis, which was diagnosed by ultrasound or laparoscopy, were recruited for this prospective, casecontrolled study. The extent of endometriosis was staged according to the American Society for Reproductive Medicine classification of endometriosis. Among these patients, 13 (surgery group) were surgically treated for endometrioma before enrollment, and 28 (non-surgery group) were diagnosed with an ovarian endometrioma by ultrasound. All 13 surgical patients underwent a conventional laparoscopic cystectomy procedure.

The exclusion criteria were as follows: $\geq 40$ years of age; body mass index (BMI) $\geq 30 \mathrm{~kg} / \mathrm{m}^{2}$; basal follicle stimulating hormone $(\mathrm{bFSH})$ concentration $\geq 12 \mathrm{mIU} / \mathrm{mL}$; polycystic ovary syndrome; cycles with the dominant FF contaminated 
with blood during oocyte retrieval; cycles with the dominant FF not yielding oocytes; other endocrine diseases (thyroid disease, diabetes mellitus, and Cushing's syndrome). The semen quality of the partners of recruited women was normal.

Within a month prior to starting the IVF stimulation, all patients underwent blood sampling to determine $\mathrm{AMH}$ levels using a standard enzyme-linked immunosorbent assay (ELISA; Beckman Coulter AMH Gen II, Brea, CA, USA; normal range $=1-8 \mathrm{ng} / \mathrm{mL}$ ). In order to avoid the effect of surgery/analog treatment on AMH levels, the time between surgery and enrollment was more than three months.

\section{Controlled ovarian stimulation and IVF}

Ovarian stimulation in all patients was initiated using the ultra-long gonadotropin-releasing hormone agonist (GnRHa) protocol, as previously described [25]. Pituitary desensitization was induced with the administration of $3.75 \mathrm{mg}$ GnRHa (Triptorelin, Ferring, Germany) on day 2 or 3 of the menstrual cycle. After 28 days, patients underwent transvaginal ultrasonographic and biochemical evaluations. Once a suitable degree of downregulation was achieved (i.e., subjects had a serum oestradiol concentration $\leq 40 \mathrm{pg} / \mathrm{mL}$, an endometrial thickness $\leq 5$ $\mathrm{mm}$, and arrested follicular development), human menopausal gonadotrophin (hMG; Menogon, Ferring GmbH, Kiel, Germany) or recombinant FSH (Puregon; Organon, Dublin, Ireland or Gonal F; Merck Serono S.p.A., Modugno, Italy) was administered with a starting dose of 150-300 IU per day based on the antral follicle count (AFC), age, and body mass index (BMI), according to standard operating procedures. Ovarian response was monitored by serial transvaginal scanning and hormonal monitoring. Gonadotrophin [12] dosage was further adjusted based on the ovarian response. When one to three leading follicles was $\geq 18 \mathrm{~mm}$ in diameter, $250 \mu \mathrm{g}$ of human chorionic gonadotropin (hCG, Ovidrel, Merck Serono S.p.A.) was administered to trigger final maturation of the oocytes. Oocyte retrieval was performed 36 $\mathrm{h}$ later. Embryos were graded on day 3 according to a 1 to 3 consensus scoring system (with 1 being the top embryos), which was based on cell size and symmetry, fragmentation, multinucleation, and blastomere number (Alpha Scientists in Reproductive Medicine and ESHRE Special Interest Group of Embryology, 2011). Two embryos were transferred 3 days later. Excess good quality embryos were frozen for subsequent transfer. The luteal phase was supported with $90 \mathrm{mg}$ of $8 \%$ progesterone gel (Crinone, Merck Serono) or $800 \mathrm{mg}$ of micronized progesterone (Utrogestan, Laboratoires Besins International, Paris, France) daily. Progesterone support was initiated on the day of oocyte retrieval and continued for 14 days; the treatment continued for another 8 weeks if a pregnancy was achieved. A clinical pregnancy was identified 4-5 weeks after oocyte retrieval by the presence of an intrauterine gestational sac and a pulsating fetal heartbeat.

\section{FF collection and detection of cytokine profiles}

FF was obtained from a single, large diameter dominant follicle during oocyte retrieval and stored at $-80^{\circ} \mathrm{C}$ until further use. FF samples that were contaminated with blood were excluded. Sixteen selected cytokines/chemokines involved in inflammatory and angiogenic pathways in FF were detected by multiplex analysis using the Milliplex Magnetic Bead assay (Millipore, Billerica, MA, USA). The assay contained granulocyte colony-stimulating factor (G-CSF), granulocyte-macrophage colony-stimulating factor (GM-CSF), interferon (IFN) $\gamma$, chemokine (C-C motif) ligand 2 (CCL2), tumor necrosis factor (TNF) $\alpha$, vascular endothelial growth factor (VEGF), CCL3, interleukin (IL) 1ß, IL-2, IL-5, IL-6, IL-8, IL-10, IL-12(p70), IL-15, and IL-18. The Luminex 200TM system and Milliplex Analyst were used for detection and analysis.

\section{Statistics}

Data were analyzed with the Statistical Program for Social Sciences (SPSS; version 18.0). Statistical comparisons were carried out using the Mann-Whitney $U$ test, chi-square test, and Student's $t$-test when appropriate. Pearson's bivariate correlation coefficient analysis was performed to determine correlations. Pearson's bivariate correlation analysis was performed to identify factors predicting IVF outcomes. A two-sided $P<0.05$ was considered statistically significant.

\section{Results}

\section{Clinical characteristics of patients and indexes of IVF treatment}

There were no significant differences in age, duration of infertility, BMI, basal FSH levels or AFC between the surgery and non-surgery groups. The $\mathrm{AMH}$ level was lower in the surgery group $(1.96 \pm 1.00 \mathrm{ng} / \mathrm{mL})$ than in the non-surgery group $(3.71 \pm 2.21 \mathrm{ng} / \mathrm{mL}, P=0.01)$. There were fewer retrieved oocytes in the surgery group than in the non-surgery group $(7.0 \pm 5.69$ vs. $12.32 \pm$ $8.60, P=0.05)$, but this difference was not statistically significant. The number, the size, and the bilaterality of the cysts did not significantly differ between the two groups (Table 1). Other IVF treatment characteristics (total Gn dose, medication used, E2 on HCG day, the number of MIloocytes, fertilization rate, high quality embryo rate overall transferred, and endometrial thickness) and the implantation and clinical pregnancy rates were similar between the two groups (Table 2). These results suggest that endometrioma surgery may potentially reduce the ovarian reserve and has little impact on the success rate of IVF. 
Table 1 Clinical characteristics for the patient groups

\begin{tabular}{llll}
\hline Parameter & $\begin{array}{l}\text { Non-surgery } \\
(n=28)\end{array}$ & $\begin{array}{l}\text { Surgery } \\
(n=13)\end{array}$ & $P$ \\
\hline Age (years) & $31.57 \pm 3.10$ & $33.85 \pm 5.47$ & 0.10 \\
Duration of infertility (years) & $3.79 \pm 2.54$ & $4.23 \pm 2.49$ & 0.60 \\
BMI (kg/m2) & $22.72 \pm 2.67$ & $21.40 \pm 2.47$ & 0.14 \\
Diameter of the cyst (cm) & & & \\
$\quad<4 \mathrm{~cm}$ & $9(32.1 \%)$ & $3(23.1 \%)$ & 0.72 \\
$\quad$ Z $4 \mathrm{~cm}$ & $19(67.9 \%)$ & $10(76.9 \%)$ & \\
Number of cysts & & & \\
$\quad$ single cyst & $17(60.7 \%)$ & $7(53.8 \%)$ & 0.68 \\
$\quad$ multiple cyst & $11(39.3 \%)$ & $6(46.2 \%)$ & \\
Endometrioma & & & \\
$\quad$ Unilateral & $22(78.6 \%)$ & $9(69.2 \%)$ & \\
$\quad$ Bilateral & $6(21.4 \%)$ & $4(31.0 \%)$ & 0.70 \\
bFSH (IU/L) & $7.08 \pm 1.74$ & $7.97 \pm 2.13$ & 0.17 \\
AMH (ng/ml) & $3.71 \pm 2.21$ & $1.96 \pm 1.00$ & $0.01^{*}$ \\
AFC & $12.15 \pm 5.51$ & $8.69 \pm 4.84$ & 0.06 \\
\hline
\end{tabular}

$B M I$ body mass index, $b F S H$ basal follicle-stimulating hormone, $A M H$ antiMüllerian hormone, $A F C$ antral follicles count. Data are expressed as mean \pm standard deviation. The $P$ values were obtained from the Student's $t$-test. The Chi-square test was used for the ratio analysis

${ }^{*} P<0.05$ was considered statistically significant

\section{Levels of cytokines in FF of women with endometrioma}

The levels of cytokines, including G-CSF, GM-CSF, IFN$\gamma$, IL-1 $\beta$, IL-2, IL-5, IL-6, IL-8, IL-10, IL-12(p70), IL-15, IL-18, TNF- $\alpha$, CCL2, CCL3, and VEGF, were determined in the FF of patients with endometrioma. The

Table 2 IVF treatment characteristics for the patient groups

\begin{tabular}{llll}
\hline Parameter & $\begin{array}{l}\text { Non-surgery } \\
(n=28)\end{array}$ & $\begin{array}{l}\text { Surgery } \\
(n=13)\end{array}$ & $P$ \\
\hline Total Gn dose (U) & $\begin{array}{l}2012.03 \pm \\
668.07\end{array}$ & $\begin{array}{l}2243.46 \pm \\
596.76\end{array}$ & 0.29 \\
medication used & $9(32.1 \%)$ & $5(38.5 \%)$ & 0.73 \\
$\quad$ rFSH & $19(67.9 \%)$ & $8(61.5 \%)$ & \\
hMG & $3628.20 \pm$ & $2399.7 \pm$ & 0.13 \\
E2 $_{2}$ on HCG day (pg/ml) & 2639.51 & 1082.21 & \\
& $12.32 \pm 8.60$ & $7.00 \pm 5.69$ & 0.05 \\
Retrieved oocytes & $9.19 \pm 6.98$ & $6.08 \pm 4.83$ & 0.16 \\
MII oocytes & $(249 / 345)$ & $(74 / 91)$ & 0.08 \\
Fertilization rate (\%) & $72.2 \%$ & $81.3 \%$ & \\
& $91.1(52 / 56)$ & $91.5(24 / 26)$ & 1.0 \\
High Quality embryo rate overall & & & \\
transferred (\%) & $10.71 \pm 1.70$ & $10.91 \pm 1.85$ & 0.65 \\
Endometrial thickness [19] & 32.6 & 26.9 & 0.79 \\
Implantation rate (\%) & 46.4 & 46.2 & 1.00 \\
Clinical pregnancy rate (\%) & &
\end{tabular}

Gn gonadotropins, E2 estradiol, HCG human chorionic gonadotropin. HMG human menopausal gonadotrophin, MII metaphase II. Data are expressed as mean \pm standard deviation. The $P$ values were obtained from the Student's $t$ test. The Chi-square test was used for the ratio analysis concentrations of IL-5 and IL-10 could not be quantified because they were below the detection level. The levels of the other cytokines are shown in radar charts (Fig. 1). Among the soluble molecules examined in this study, chemokines and growth factors (Fig. 1a) and a few are inflammatory cytokines (Fig. 1b) were the primary components found in the follicular fluid of patients with endometriosis.

\section{Impact of lesion removal on the levels of cytokines in follicular fluid}

To explore whether or not lesion removal influenced the cytokine profiles in FF, we determined the cytokine levels in FF obtained from patients in the surgery (S, $n=$ 13 ) and non-surgery (NS, $n=28)$ groups. The concentrations of the 16 cytokines in FF obtained from the two different groups are presented in Fig. 2. We also assessed differences in cytokine levels between the two groups. As shown in Fig. 3, the levels of chemokines (CCL2, CCL3, and CXCL8) and growth factors (G-CSF, GMCSF, and VEGF) did not significantly differ between the two groups. In addition, the levels of inflammatory cytokines (IFN- $\gamma$, IL-1 $\beta$, IL-2, IL-6, IL-12(p70), IL-15, and TNF- $\alpha$ ) did not differ between the two groups. However, the concentration of IL-18 in the FF of patients in the surgery group was significantly higher than in the nonsurgery group $(213.92 \pm 74.30 \mathrm{pg} / \mathrm{mL}$ vs. $145.12 \pm 74.20$ $\mathrm{pg} / \mathrm{mL}, P=0.01)$. Thus, surgical removal of endometriotic lesions might stimulate the production of IL-18 in the FF of women with endometriosis.

\section{Relationship between cytokine levels in follicular fluid and IVF outcomes}

Because the levels of IL-18 in FF differed between patients in the surgery and non-surgery groups, we further studied the correlation between IL-18 level and oocyte and embryo quality using Pearson's bivariate correlation analysis. The levels of IL-18 in the FF samples did not correlate with these cycle parameters (Table 3).

\section{Discussion}

In the present study, we investigated the effect of endometrioma cystectomy on the cytokine profiles in FF and IVF outcomes. The findings demonstrate that surgical treatment prior to IVF had a negative impact on the ovarian reserve and did not improve the clinical pregnancy rate of IVF in women with endometriosis. In addition, our results suggest that surgery for endometriomas might promote increased production of IL-18; however, we failed to find a correlation between IL-18 level and oocyte or embryo quality. Our results add to our understanding of the effect of surgical treatment on the intrafollicular microenvironment in women with endometriosis. The surgery for endometriomas prior to 


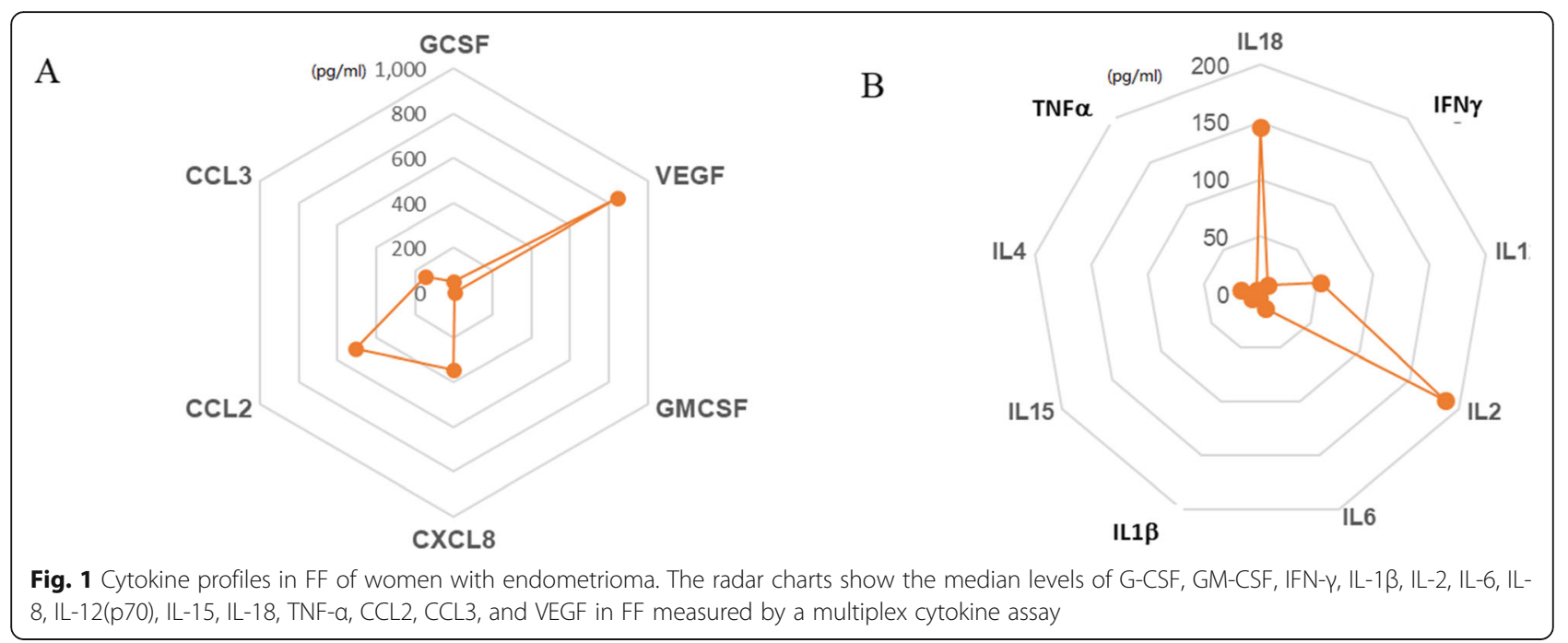

performing artificial reproduction technology (ART) procedures remain controversial. Barri et al. (2010) found that a combined strategy of endoscopic surgery and subsequent IVF led to a significantly higher clinical pregnancy rate than that obtained with IVF alone [26]. Candiani et al. (2018) demonstrated a significant improvement in the AFC of the surgically treated ovary after $\mathrm{CO}_{2}$ laser vaporization [27]. However, conflicting results have been reported. Wahd et al. (2014) found that women receiving intracytoplasmic sperm injections following surgery for ovarian endometrioma had poorer clinical outcomes and lower rates of live births compared to women with endometriosis (without previous surgery) and women with no endometriosis [28]. In addition, several studies have found that surgical removal of endometriotic lesions prior to ART treatment does not improve reproductive outcomes, but rather decreases the ovarian reserve $[29,30]$. For example, Ata et al. (2017) found that women with endometriomas had a lower ovarian reserve than age-matched controls, and this reserve was further reduced by surgical excision of endometriomas [31]. Several studies have since confirmed that the ovarian reserve damage and the IVF response after surgery was related to the size of the ovarian cysts and the presence of bilateral endometrioma [32-35].

In the current study, we showed that surgical treatment of endometriomas before IVF did not benefit the clinical outcome of IVF and may have decreased the ovarian reserve, serum AMH levels, and AFC. All the surgery-group patients in our study underwent a conventional laparoscopic cystectomy procedure. This type of surgery may decrease the ovarian reserve because it may remove the ovarian cortex and modify the ovarian arterial blood flow [36]. Our results are also consistent with the 2014 ESHRE guidelines recommending clinicians only consider performing cystectomy for endometriomas larger than $3 \mathrm{~cm}$ prior to IVF if necessary to improve pelvic pain or to facilitate access to the follicles

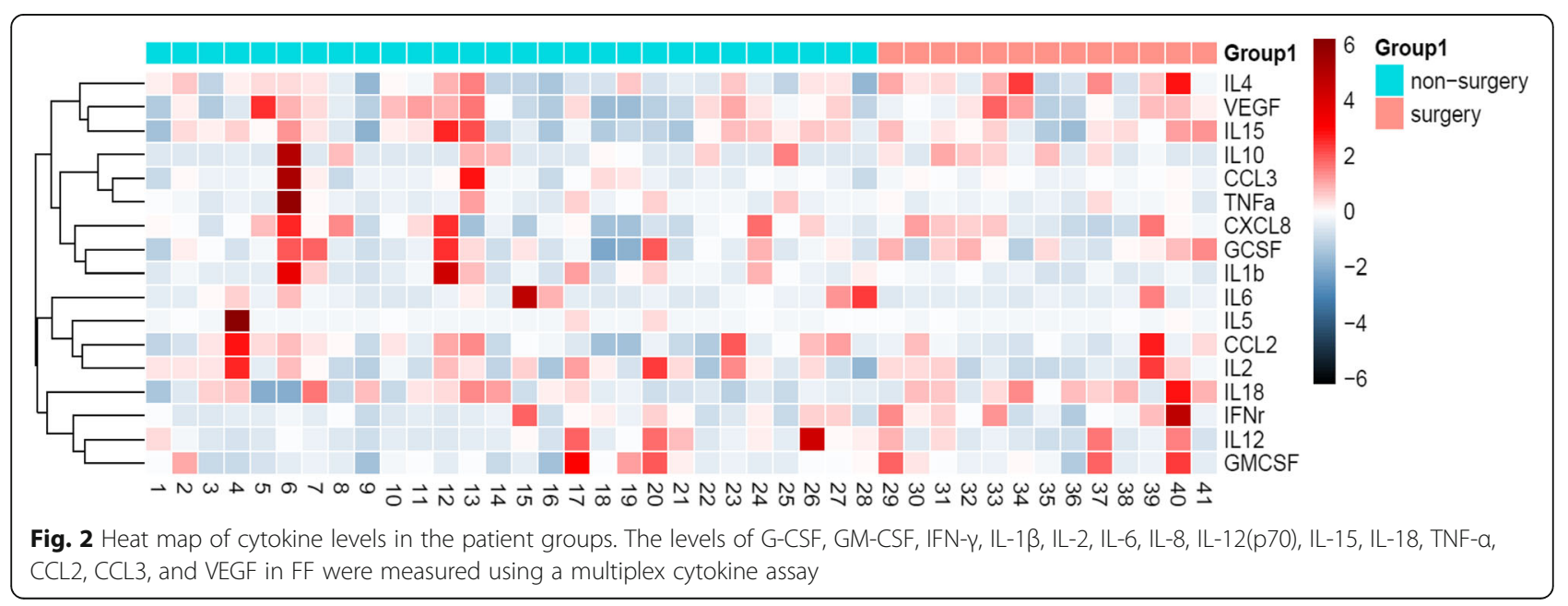




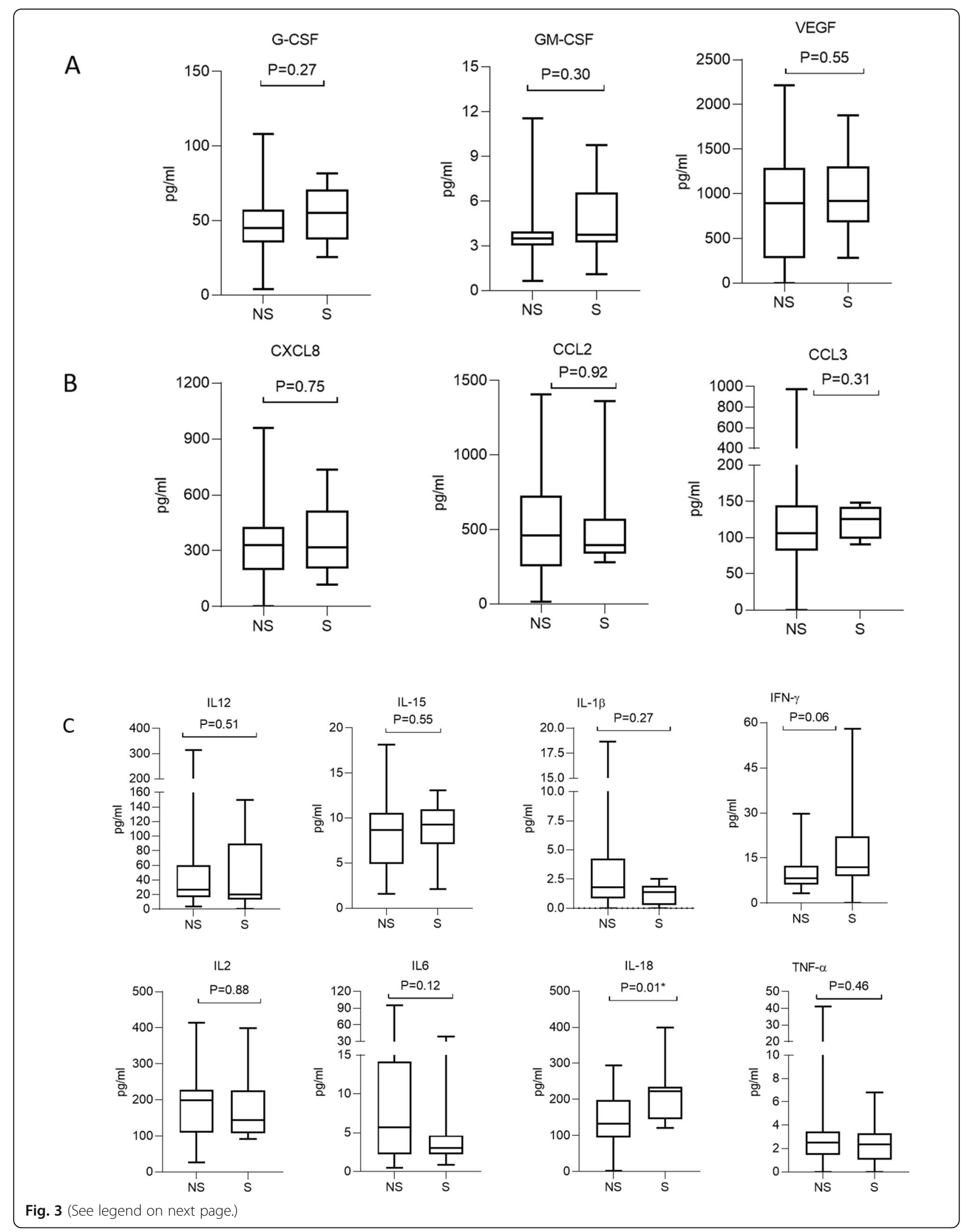


(See figure on previous page.)

Fig. 3 Concentrations of cytokines in the FF of patients. The cytokine levels in the FF from 13 patients in the surgery (S) group and 28 patients in the non-surgery (NS) group were analyzed. (a) Growth factors (G-CSF, GM-CSF, and VEGF); (b) Chemokines (CCL2, CCL3, and CXCL8); (c) Inflammatory factors (IFN- $\gamma, \mathrm{IL}-1 \beta, \mathrm{IL}-2, \mathrm{IL}-6, \mathrm{IL}-12(\mathrm{p} 70)$, IL-15, IL-18, and TNF-a). The horizontal lines in the box plots represent the median and the 25th and 75th percentiles. The $P$ values were obtained from the Mann-Whitney $U$ test

during oocyte retrieval [37]. Although there was a reduction in the serum AMH level following endometrioma surgery, the pregnancy rates were no different from patients with untreated endometriomas. Porpora et al. (2014) observed that after laparoscopic treatment of ovarian endometriomas, the uterine arterial flow was significantly improved, which seems to have increased the probability of achieving pregnancy [38]. This outcome will be further investigated in our future study.

According to the literature, the presence of endometriomas produces a cytokine imbalance in the peritoneal environment. Additionally, altered production of some cytokines and inflammatory factors in the FF of women with endometriosis has been observed. Monsanto et al. (2016) found that ectopic lesions were major drivers of systemic inflammation in endometriosis and that endometriotic lesion removal significantly altered the inflammatory profile both locally and systemically in women with endometriosis [39]. However, little information is available regarding the alterations of these factors in the FF. In the present study, we found that the levels of chemokines and growth factors were relatively higher than those of inflammatory cytokines in the FF of women with endometriosis-associated infertility. When comparing the surgery group to the non-surgery group, no significant differences were observed in the levels of chemokines, growth factors, or inflammatory cytokines except for IL-18; the levels of IL-18 were significantly higher in patients who underwent surgery prior to IVF treatment.

Our findings are consistent with the relationship between IL-18 and endometriosis identified in other studies. Arici et al. (2003) found measurable levels of IL18 in the peritoneal fluid of patients receiving $\mathrm{GnRH}$ agonists for endometriosis to be significantly higher than those of the control group-patients with endometriosis without treatment [40]. Luo et al. (2006) reported the down-regulation of IL-18 mRNA expression in the ectopic and eutopic endometria of women with endometriosis [41]. In addition, the concentration of IL-18 in the peritoneal fluid was significantly lower in patients with endometriosis than in those without endometriosis [42], suggesting IL-18 might play a pathogenic role in the formation of endometriosis.

Cytokines play an essential but complex role as local regulators of ovarian function, and this role is an area of active investigation. IL-18, initially described as an interferon (IFN) $\gamma$ inducing factor, plays a central role in the inflammatory cascade and in the process of innate and acquired immunity. It is involved not only in Th1 and NK cell activation, but also in Th2 and Th17 modulation, as well as macrophage activation. IL-18 is known to induce cytokines that are important for both folliculogenesis and ovulation, including IL-1 $\beta$, TNF- $\alpha$, and IFN$\gamma$ [43]. Salmassi et al. (2017) found that the follicular granulosa cells are the major source of IL-18 and the site of IL-18 receptor expression [43]. The role of IL-18 in oocytes is controversial. Sarapik et al. (2012) demonstrated that IL-18 levels were positively correlated with the number of retrieved oocytes and implantation success in women with different etiologies of infertility [17]. However, conflicting results have been reported. Radwan et al. (2013) did not find any correlation between IL-18 and the number of mature MII oocytes or good-quality embryos in women with "tubal obstruction" subjected to IVF [44]. In agreement with the study of Radwan et al. (2013), we also failed to find a correlation between IL-18 and oocytes or embryo quality in women with endometriosis-associated infertility. Different etiologies of infertility may be one reason for the different outcomes. We therefore hypothesize that surgical intervention might facilitate the expression of IL-18, which may be beneficial to the treatment of endometriosis, but does not improve the quality of oocytes.

Our study has several limitations. Due of the proportion of endometriomas in IVF and the frequently complicated clinical manifestations, it is challenging to collect large numbers of samples from our center. The relativity small sample size is a major limitation of this study, which did not allow us to draw definitive conclusions. Second, ours was a retrospective case-controlled study, and the patients had already undergone surgical excision of their endometriomas before presenting for IVF treatment. As a result, we could not compare their

Table 3 Pearson's correlation coefficients between levels of cytokines in FF and IVF outcomes

\begin{tabular}{|c|c|c|c|c|c|c|}
\hline & \multicolumn{2}{|c|}{ No. of mature oocytes/no. of total oocytes (\%) } & \multicolumn{2}{|c|}{ Fertilization rate (\%) } & \multicolumn{2}{|c|}{ No. of high-quality embryos/no. of embryos } \\
\hline & $r$ & $P$ & $r$ & $\mathrm{P}$ & $r$ & $P$ \\
\hline $\mathrm{IL}-18$ & -0.05 & 0.75 & -0.02 & 0.89 & 0.09 & 0.59 \\
\hline
\end{tabular}


preoperative and postoperative data. Further prospective randomized controlled trials are required.

Taken together, our findings suggest that endometrioma surgery may potentially reduce the ovarian reserve and has little impact on the success rate of IVF. Ovarian endometriomas are not associated with cytokine profiles in $\mathrm{FF}$ from infertile women scheduled for IVF, and they are not likely to affect the quality of the oocyte and embryo as a result of an inflammatory mechanism.

\section{Abbreviations}

AFC: Antral follicle count; AMH: Anti-Müllerian hormone.; ART: Artificial reproduction technology; bFSH: Basal follicle stimulating hormone; BMI: Body mass index; CCL: Chemokine (C-C motif) ligand; CXCL: Chemokine (C-X-C motif) ligand; FF: Follicular fluid; G-CSF: Granulocyte colony-stimulating factor; GM-CSF: Granulocyte-macrophage colony-stimulating factor; Gn: Gonadotrophin; GnRHa: Gonadotropin-releasing hormone agonist; IFN: Interferon; IL: interleukin; IVF: in vitro fertilization; NK: cytotoxic effect of natural killer; TNF: Tumor necrosis factor; VEGF: Vascular endothelial growth factor

\section{Acknowledgements}

The authors express their appreciation to all of the patients for their participation in this study.

\section{Authors' contributions}

$\mathrm{YL}$ designed the study, collected the samples, acquired, analyzed and interpreted the data, and drafted the manuscript; $X Y$ analyzed the data and drafted the manuscript; $Y L, Y L$ and $L L$ recruited the patients and analyzed the data; and Shuyu Wang conceived and designed the research, supervised the research, and made critical revisions of the manuscript for important intellectual content. All authors read and approved the final manuscript.

\section{Funding}

This work was supported by a grant from the National Natural Science Foundation of China (No.81601541) to Ying Li and a Beijing Municipal Administration of Hospitals Clinical Medicine Development of special funding support (ZYLX201830) provided to Xiaokui Yang.

\section{Availability of data and materials}

The datasets used and/or analyzed during the current study are available from the corresponding author on reasonable request.

\section{Ethics approval and consent to participate}

Ethical approval (registration number 2017-KY-002-01) was received from the local Institutional Ethics Committee and written informed consent was obtained from all participants.

\section{Consent for publication}

Not applicable.

\section{Competing interests}

All authors declare that there is no conflict of interest that could be perceived as prejudicing the impartiality of the research reported.

Received: 25 June 2019 Accepted: 21 September 2019

Published online: 21 October 2019

\section{References}

1. Vannuccini S, Clifton VL, Fraser IS, Taylor HS, Critchley H, Giudice LC, et al. Infertility and reproductive disorders: impact of hormonal and inflammatory mechanisms on pregnancy outcome. Hum Reprod Update. 2016;22(1):104-15.

2. Hsiao KY, Wu MH, Tsai SJ. Epigenetic regulation of the pathological process in endometriosis. Reprod Med Biol. 2017;16(4):314-9.

3. Practice Committee of the American Society for Reproductive Medicine. Endometriosis and infertility: a committee opinion. Fertil Steril. 2012:98(3): 591-8.
4. Macer ML, Taylor HS. Endometriosis and infertility: a review of the pathogenesis and treatment of endometriosis-associated infertility. Obstet Gynecol Clin N Am. 2012;39(4):535-49.

5. Choi YS, Cho S, Seo SK, Park JH, Kim SH, Lee BS. Alteration in the intrafollicular thiol-redox system in infertile women with endometriosis. Reproduction (Cambridge, England). 2015;149(2):155-62.

6. Riccio L, Santulli P, Marcellin L, Abrao MS, Batteux F, Chapron C. Immunology of endometriosis. Best Pract Res Clin Obstet Gynaecol. 2018;50:39-49.

7. Bilotas MA, Olivares CN, Ricci AG, Baston Jl, Bengochea TS, Meresman GF, et al. Interplay between endometriosis and pregnancy in a mouse model. PLoS One. 2015;10(4):e0124900.

8. Ahn SH, Khalaj K, Young SL, Lessey BA, Koti M, Tayade C. Immuneinflammation gene signatures in endometriosis patients. Fertil Steril. 2016; 106(6):1420-31.e7.

9. Jorgensen $H$, Hill AS, Beste MT, Kumar MP, Chiswick E, Fedorcsak P, et al. Peritoneal fluid cytokines related to endometriosis in patients evaluated for infertility. Fertil Steril. 2017;107(5):1191-9.e2.

10. Szymanowski K, Niepsuj-Binias J, Dera-Szymanowska A, Wolun-Cholewa M Yantczenko A, Florek E, et al. An influence of immunomodulation on Th1 and Th2 immune response in endometriosis in an animal model. Biomed Res Int. 2013;2013:849492.

11. Izumi G, Koga K, Takamura M, Makabe T, Satake E, Takeuchi A, et al. Involvement of immune cells in the pathogenesis of endometriosis. J Obstet Gynaecol Res. 2018;44(2):191-8.

12. Matteo M, Cicinelli E, Neri M, Carrubba R, Carpagnano FA, Romeo F, et al. Pro-inflammatory M1/Th1 type immune network and increased expression of TSG-6 in the eutopic endometrium from women with endometriosis. Eur J Obstet Gynecol Reprod Biol. 2017;218:99-105.

13. Singh AK, Dutta M, Chattopadhyay R, Chakravarty B, Chaudhury K Intrafollicular interleukin-8, interleukin-12, and adrenomedullin are the promising prognostic markers of oocyte and embryo quality in women with endometriosis. J Assist Reprod Genet. 2016;33(10):1363-72.

14. Sanchez AM, Vanni VS, Bartiromo L, Papaleo E, Zilberberg E, Candiani M, et al. Is the oocyte quality affected by endometriosis? A review of the literature. J Ovarian Res. 2017;10(1):43.

15. Zhang QF, Chen GY, Liu Y, Huang HJ, Song YF. Relationship between resistin and IL-23 levels in follicular fluid in infertile patients with endometriosis undergoing IVF-ET. Adv Clin Exp Med. 2017;26(9):1431-5.

16. Song Y, Liu J, Qiu Z, Chen D, Luo C, Liu X, et al. Advanced oxidation protein products from the follicular microenvironment and their role in infertile women with endometriosis. Exp Ther Med. 2018;15(1):479-86.

17. Sarapik A, Velthut A, Haller-Kikkatalo K, Faure GC, Bene MC, de Carvalho BM, et al. Follicular proinflammatory cytokines and chemokines as markers of IVF success. Clin Dev Immunol. 2012:2012:606459.

18. Muteshi CM, Ohuma EO, Child T, Becker CM. The effect of endometriosis on live birth rate and other reproductive outcomes in ART cycles: a cohort study. Human Reprod Open. 2018;2018(4):hoy016.

19. Senapati S, Sammel MD, Morse C, Barnhart KT. Impact of endometriosis on in vitro fertilization outcomes: an evaluation of the Society for Assisted Reproductive Technologies Database. Fertil Steril. 2016;106(1):164-71.e1.

20. Singh N, Lata K, Naha M, Malhotra N, Tiwari A, Vanamail P. Effect of endometriosis on implantation rates when compared to tubal factor in fresh non donor in vitro fertilization cycles. J Human Reprod Sci. 2014; 7(2):143-7.

21. Miravet-Valenciano J, Ruiz-Alonso M, Gomez E, Garcia-Velasco JA. Endometrial receptivity in eutopic endometrium in patients with endometriosis: it is not affected, and let me show you why. Fertil Steril. 2017;108(1):28-31.

22. Yang C, Geng Y, Li Y, Chen C, Gao Y. Impact of ovarian endometrioma on ovarian responsiveness and IVF: a systematic review and meta-analysis. Reprod BioMed Online. 2015;31(1):9-19.

23. Hamdan M, Dunselman G, Li TC, Cheong Y. The impact of endometrioma on IVF/ICSI outcomes: a systematic review and meta-analysis. Hum Reprod Update. 2015;21(6):809-25.

24. Miller JE, Ahn SH, Monsanto SP, Khalaj K, Koti M, Tayade C. Implications of immune dysfunction on endometriosis associated infertility. Oncotarget. 2017:8(4):7138-47.

25. Liang Y, Han J, Jia C, Ma Y, Lan Y, Li Y, et al. Effect of endometrial injury on secretion of endometrial cytokines and IVF outcomes in women with unexplained subfertility. Mediat Inflamm. 2015;2015:757184. 
26. Barri PN, Coroleu B, Tur R, Barri-Soldevila PN, Rodriguez I. Endometriosisassociated infertility: surgery and IVF, a comprehensive therapeutic approach. Reprod BioMed Online. 2010;21 (2):179-85.

27. Candiani M, Ottolina J, Posadzka E, Ferrari S, Castellano LM, Tandoi I, et al. Assessment of ovarian reserve after cystectomy versus 'one-step' laser vaporization in the treatment of ovarian endometrioma: a small randomized clinical trial. Human Reprod (Oxford, England). 2018;33(12):2205-11.

28. Wahd SA, Alalaf SK, Al-Shawaf T, Al-Tawil NG. Ovarian reserve markers and assisted reproductive technique (ART) outcomes in women with advanced endometriosis. Reprod Biol Endocrinol. 2014;12:120.

29. Cecchino GN, Garcia-Velasco JA. Endometrioma, fertility, and assisted reproductive treatments: connecting the dots. Curr Opin Obstet Gynecol. 2018:30(4):223-8.

30. Cranney R, Condous G, Reid S. An update on the diagnosis, surgical management, and fertility outcomes for women with endometrioma. Acta Obstet Gynecol Scand. 2017;96(6):633-43.

31. Ata B, Mumusoglu S, Aslan K, Seyhan A, Kasapoglu I, Avci B, et al. Which is worse? A comparison of ART outcome between women with primary or recurrent endometriomas. Human reproduction (Oxford, England). 2017: 32(7):1539.

32. Younis JS, Shapso N, Fleming R, Ben-Shlomo I, Izhaki I. Impact of unilateral versus bilateral ovarian endometriotic cystectomy on ovarian reserve: a systematic review and meta-analysis. Hum Reprod Update. 2019:25(3):375-91.

33. Tang Y, Chen SL, Chen X, He YX, Ye DS, Guo W, et al. Ovarian damage after laparoscopic endometrioma excision might be related to the size of cyst. Fertil Steril. 2013;100(2):464-9.

34. Henes M, Engler T, Taran FA, Brucker S, Rall K, Janz B, et al. Ovarian cyst removal influences ovarian reserve dependent on histology, size and type of operation. Women's Health (London, England). 2018;14: 1745506518778992

35. Coccia ME, Rizzello F, Barone S, Pinelli S, Rapalini E, Parri C, et al. Is there a critical endometrioma size associated with reduced ovarian responsiveness in assisted reproduction techniques? Reprod BioMed Online. 2014:29(2):259-66.

36. Geber S, Ferreira DP, Spyer Prates LF, Sales L, Sampaio M. Effects of previous ovarian surgery for endometriosis on the outcome of assisted reproduction treatment. Reprod BioMed Online. 2002;5(2):162-6.

37. Dunselman GA, Vermeulen N, Becker C, Calhaz-Jorge C, D'Hooghe T, De Bie $B$, et al. ESHRE guideline: management of women with endometriosis. Human Reprod (Oxford, England). 2014;29(3):400-12.

38. Porpora MG, Tomao F, Manganaro L, Yazdanian D, Fuggetta E, Piccioni MG, et al. Impaired uterine artery flow associated with the presence of ovarian endometrioma: preliminary results of a prospective study. J Ovarian Res. 2014;7:1.

39. Monsanto SP, Edwards AK, Zhou J, Nagarkatti P, Nagarkatti M, Young SL, et al. Surgical removal of endometriotic lesions alters local and systemic proinflammatory cytokines in endometriosis patients. Fertil Steril. 2016; 105(4):968-77.e5.

40. Arici A, Matalliotakis I, Goumenou A, Koumantakis G, Vassiliadis S, Mahutte NG. Altered expression of interleukin-18 in the peritoneal fluid of women with endometriosis. Fertil Steril. 2003;80(4):889-94.

41. Luo Q, Ning W, Wu Y, Zhu X, Jin F, Sheng J, et al. Altered expression of interleukin-18 in the ectopic and eutopic endometrium of women with endometriosis. J Reprod Immunol. 2006;72(1-2):108-17.

42. Zhang $X$, Lin J, Qian Y, Deng L. Decreased levels of interleukin-18 in peritoneal fluid but not in serum of patients with endometriosis. Fertil Steril. 2004;81(5):1229-34.

43. Salmassi A, Fattahi A, Nouri M, Hedderich J, Schmutzler AG. Expression of mRNA and protein of IL-18 and its receptor in human follicular granulosa cells. J Endocrinol Investig. 2017:40(4):447-54.

44. Radwan P, Radwan M, Polac I, Lewkowicz P, Wilczynski JR. IL-18 and IL-18 binding protein concentration in ovarian follicular fluid of women with "tubal factor" subjected to in vitro fertilization. Postepy higieny i medycyny doswiadczalnej (Online). 2013:67:463-70.

\section{Publisher's Note}

Springer Nature remains neutral with regard to jurisdictional claims in published maps and institutional affiliations.

\section{Ready to submit your research? Choose BMC and benefit from:}

- fast, convenient online submission

- thorough peer review by experienced researchers in your field

- rapid publication on acceptance

- support for research data, including large and complex data types

- gold Open Access which fosters wider collaboration and increased citations

- maximum visibility for your research: over $100 \mathrm{M}$ website views per year

At BMC, research is always in progress.

Learn more biomedcentral.com/submissions 